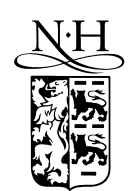

ELSEVIER

Applied Surface Science 141 (1999) 201-209

\title{
Carbon nanotubes: nanomechanics, manipulation, and electronic devices
}

\author{
Ph. Avouris * , T. Hertel, R. Martel, T. Schmidt, H.R. Shea, R.E. Walkup \\ IBM Research Division, T.J. Watson Research Center, Yorktown Heights, NY 10598, USA
}

Received 29 May 1998; accepted 2 July 1998

\begin{abstract}
Carbon nanotubes are novel materials with unique electrical and mechanical properties. Here we present results on their atomic structure and mechanical properties in the adsorbed state, on ways to manipulate individual nanotubes, on their electrical properties and, finally, on the fabrication and characteristics of nanotube-based electron devices. Specifically, atomic force microscopy (AFM) and molecular mechanics simulations are used to investigate the effects of van der Waals interactions on the atomic structure of adsorbed nanotubes. Both radial and axial structural deformations are identified and the interaction energy itself is obtained from the observed deformations. The conditions under which the structure of a nanotube will adjust to the topography of the substrate are defined. We show that the strong substrate-nanotube interaction allows the manipulation of both the position and shape of individual nanotubes at inert surfaces using the AFM. AFM manipulation is then utilized to position individual nanotubes on electrical pads so that their electrical characteristics can be evaluated. We demonstrate the operation of a field-effect transistor based on a single semiconducting nanotube and of a single-electron transistor using a nanotube bundle as Coulomb island. Finally, conducting nanotubes are employed as tips for AFM lithography. (C) 1999 Elsevier Science B.V. All rights reserved.
\end{abstract}

PACS: 85.65; 85.30.T; 71.24; 61.16.C

Keywords: Carbon nanotubes; Molecular manipulation; Molecular electron devices

\section{Introduction}

Carbon nanotubes are an interesting class of nanostructures which can be thought of as arising from the folding of a layer of graphite (a graphene sheet) to form a hollow cylinder composed of carbon hexagons. A number of techniques including discharges between carbon electrodes, laser vaporiza-

\footnotetext{
* Corresponding author. Tel.: + 1-914-945-2722; Fax: + 1-914945-4531; E-mail: avouris@us.ibm.com
}

tion of carbon, and thermal decomposition of hydrocarbons have been used to prepare these materials $[1,2]$. Depending on the width of the graphene sheet and the way it is folded a variety of different nanotube structures can be formed. As shown in Fig. 1, to describe these structures a chiral vector is defined as $\boldsymbol{C}=n \boldsymbol{a}+m \boldsymbol{b}=(n, m)$, where $\boldsymbol{a}$ and $\boldsymbol{b}$ are unit vectors of the two-dimensional graphene sheet and $n, m$ are integers, along with a chiral angle $\theta$, which is the angle of the chiral vector with respect to the zig-zag direction of the graphene sheet [1]. When the graphene sheet is rolled up to form a particular 


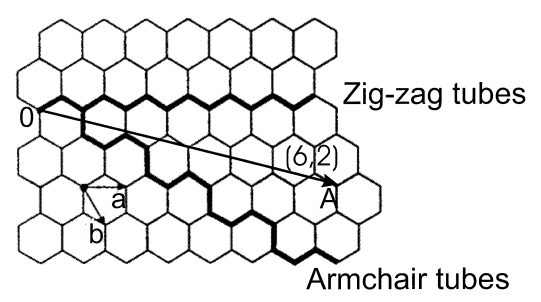

Fig. 1. A graphene sheet with unit vectors $\boldsymbol{a}$ and $\boldsymbol{b}$. A nanotube can be formed by folding the sheet so that the end points $\mathrm{O}$ and $\mathrm{A}$ of the vector $\boldsymbol{C}=n \boldsymbol{a}+m \boldsymbol{b}=(n, m)$ coincide. When $n=m$, the ends of the tube have a meander-like structure and the tube is called an armchair tube. When $n=0$, the ends of the nanotube have a zig-zag structure. Nanotubes with arbitrary values of $n$ and $m$ have a chiral structure.

nanotube, the two ends of the chiral vector are joined, and the chiral vector forms the circumference of the nanotube. The unique electronic properties of the nanotube are the result of the quantum confinement of the electrons along its circumference. The one-dimensional electronic structure of a nanotube can be predicted on the basis of the two-dimensional electronic structure of graphite [1,3]. Thus, all $(n, n)$ nanotubes are predicted to be metallic, and the same is true for $(n, m)$ tubes when $|n-m|=3 i$, where $i$ is an integer. The electronic structure of all other nanotubes has an energy gap and the tubes are semiconductors. Recent scanning-tunneling spectroscopy experiments have verified these predictions [4,5]. Finally, we note that carbon nanotubes can be made of a single layer of carbon atoms (single-walled tubes) or many (multi-walled tubes).

The interesting electrical properties described above coupled with their high mechanical strength $[6,7]$ give a unique character to these materials. A great number of applications have been proposed [1]. In particular, nanotubes appear very promising as one-dimensional nanowires and as switching elements in novel nanoelectronic devices.

Here we discuss a number of issues involving the structure, interactions, manipulation and applications of nanotubes. Specifically, we present results indicating that the van der Waals interaction of nanotubes with the substrate on which they are deposited on can be quite strong, inducing both axial and radial distortions of the atomic structure of nanotubes. The van der Waals binding energy itself can be obtained by measuring the extent of these distortions. The deformations reduce the symmetry of the nanotube structure and can modify the electronic and mechanical properties [8,9]. Furthermore, the strong nanotube-substrate interaction, by stabilizing highly strained configurations, makes possible the manipulation of not only the position but also the shape of individual nanotubes at room temperature using the tip of an AFM. In this way, nanotubes can be positioned on electrical contacts such that electrical transport through single nanotubes can be measured. Most importantly, it is shown that transport through semiconducting nanotubes can be modulated to generate carbon nanotube field-effect transistors. Single-electron transistor operation is demonstrated as well. Finally, we show that the metallic character of nanotubes allows their use as electrodes for the local electrochemical modification (oxidation) of surfaces. Details on the experimental and computational techniques used can be found in the locally cited references.

\section{Nanomechanical properties of nanotubes}

As mentioned in the introduction, the electronic properties of carbon nanotubes are derived from those of graphite on the basis of symmetry related arguments. Unlike free nanotubes, however, nanotubes supported on a solid substrate may be distorted both axially and radially. Fig. 2 shows two non-contact AFM images of overlapping multi-wall nanotubes dispersed from a dichloroethane solution on a H-passivated silicon surface. In this case, the nanotubes are expected to interact with the inert substrate by van der Waals forces. The images clearly show that the upper tubes bend around the lower ones. As we have discussed in detail elsewhere [8,9], these distortions arise form the tendency to increase the area of contact between the upper tubes and the substrate so as to increase their adhesion energy. Counteracting this tendency is the increase in strain energy that follows the increased curvature of the upper tubes. The total energy of the system can be expressed as an integral of the strain energy $U(c)$ and the adhesion energy $V(z)$ over the entire tube profile: $E=\int[U(c)+V(z(x))] \mathrm{d} x$. Here, $c$ is the 

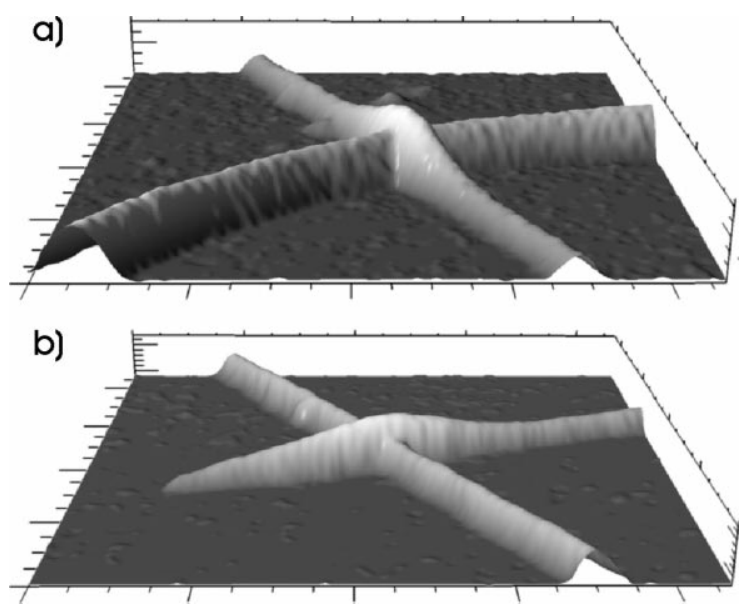

Fig. 2. Atomic force microscope (AFM) non-contact mode images of two overlapping multi-wall nanotubes. The upper tubes are seen to wrap around the lower ones which are slightly compressed. The size of image (a) is $330 \mathrm{~nm}$ and that of (b) is 500 nm.

local tube curvature and $V(z(x))$ the nanotube-substrate interaction potential at a distance $z$ above the surface. Using the measured Young's modulus for multi-walled nanotubes [6] and by fitting to the experimentally observed nanotube profile, one can estimate the binding energy from the observed distortion. In this way, we obtain a binding energy of $\sim 0.8 \mathrm{eV} / \AA$ for nanotubes with a diameter of about $100 \AA$. Thus, van der Waals binding energies, which for individual atoms or molecules are weak (typically $0.1 \mathrm{eV}$ ), can be quite strong for mesoscopic systems such as the carbon nanotubes. High binding energies imply that strong forces are exerted by nanotubes on underlying surface features such as steps, defects, or other nanotubes. For example, the force leading to the compression of the lower tubes in Fig. 2a is estimated to be as high as $35 \mathrm{nN}$.

In addition to the experimental study of the distortions induced by van der Waals forces, we have performed molecular mechanics simulations of the same phenomena [9]. The MM3 alkene force-field was used to model the intra-tube atomic interactions, while the van der Waals interaction parameters were obtained by summing atomic van der Waals interactions for $\mathrm{sp}^{2}$ hybridized carbon atoms interacting with a graphite slab [9]. Fig. 3a and b show the distortions arising when two single-walled $(10,10)$ nanotubes cross each other. In addition to their axial distortion, the two nanotubes are seen to have a distorted, non-circular cross-section in the overlap region. The results of molecular mechanics calculations of the radial distortions of single-walled nanotubes due to van der Waals interaction with a graphite surface are shown in Fig. 3c. It is found that the adhesion forces tend to flatten the bottom of the tubes so as to increase the area of contact. Concomitant with this increase in adhesion is an increase of the curvature of the tube and therefore a rise in strain energy. The overall shape is dictated by the optimization of these two opposing trends. Small diameter tubes that already have a high curvature resist further distortion, while large tubes, such as the $(40,40)$ tube, flatten out and increase considerably their binding energy [by $115 \%$ in the case of the $(40,40)$ tube]. In the case of multi-wall tubes, we find that, as the number of shells increases, the

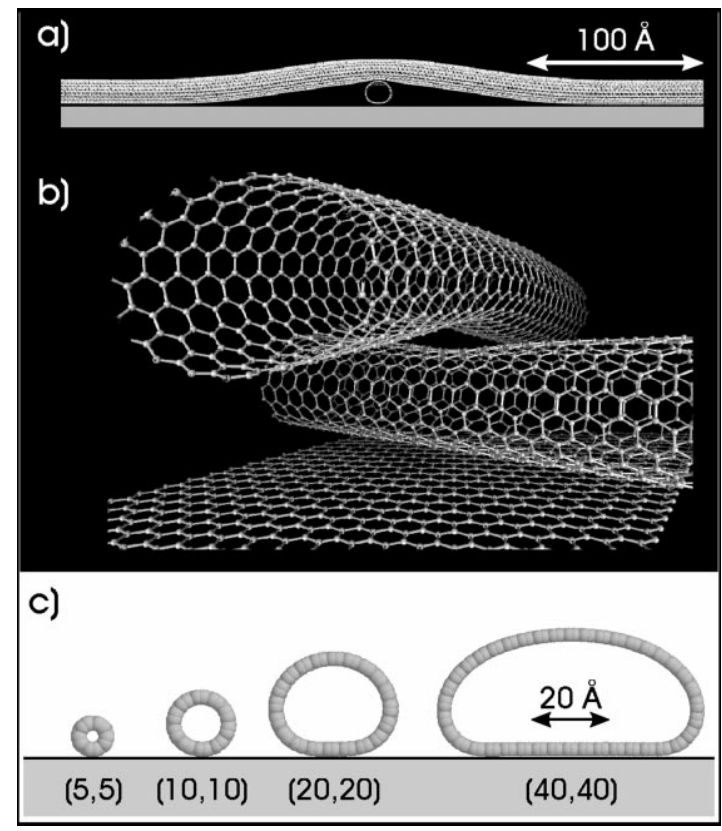

Fig. 3. Molecular mechanics calculations on the axial and radial deformations of single-wall carbon nanotubes. (a) Axial deformation resulting from the crossing of two $(10,10)$ nanotubes. (b) Perspective close up of the same crossing showing that both tubes are deformed near the contact region. The force acting on the lower tube is about $5 \mathrm{nN}$. (c) Computed radial deformations of single-wall nanotubes on graphite. 
overall gain in adhesion energy due to distortion decreases as a result of the rapidly increasing strain energy [9].

From the AFM results and the molecular mechanics calculations, we conclude that carbon nanotubes in general tend to adjust their structure to follow the surface morphology of the substrate. We can define a critical radius of surface curvature $R_{\mathrm{c}}$ above which the nanotube can follow the surface structure or roughness. Given that the strain energy varies more strongly with tube diameter than the adhesion energy, the critical radius is a function of the tube diameter. Fig. 4 shows the approximate variation of $R_{\mathrm{c}}$ with $d$ for $(5,5)$ and $(10,10)$ single-wall and larger multi-wall tubes based on binding and strain energies computed with molecular mechanics.

The van der Waals forces play an important role not only in the interaction of the nanotubes with the substrate but also in their mutual interaction. The different shells of a multi-walled tube interact by van der Waals forces; single-walled tubes form ropes for the same reason. Recently, we have found a way to convert short ropes of single-wall nanotubes into nanotube rings [10]. An example is shown in Fig. 5.
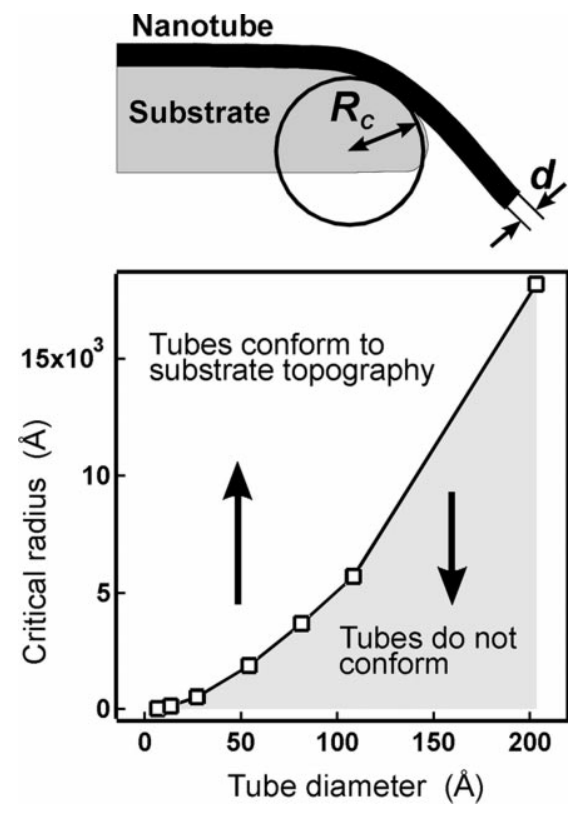

Fig. 4. Criteria determining whether a nanotube with a particular diameter $d$ conforms to the local topography of the substrate characterized by a radius of curvature $R_{\mathrm{c}}$.
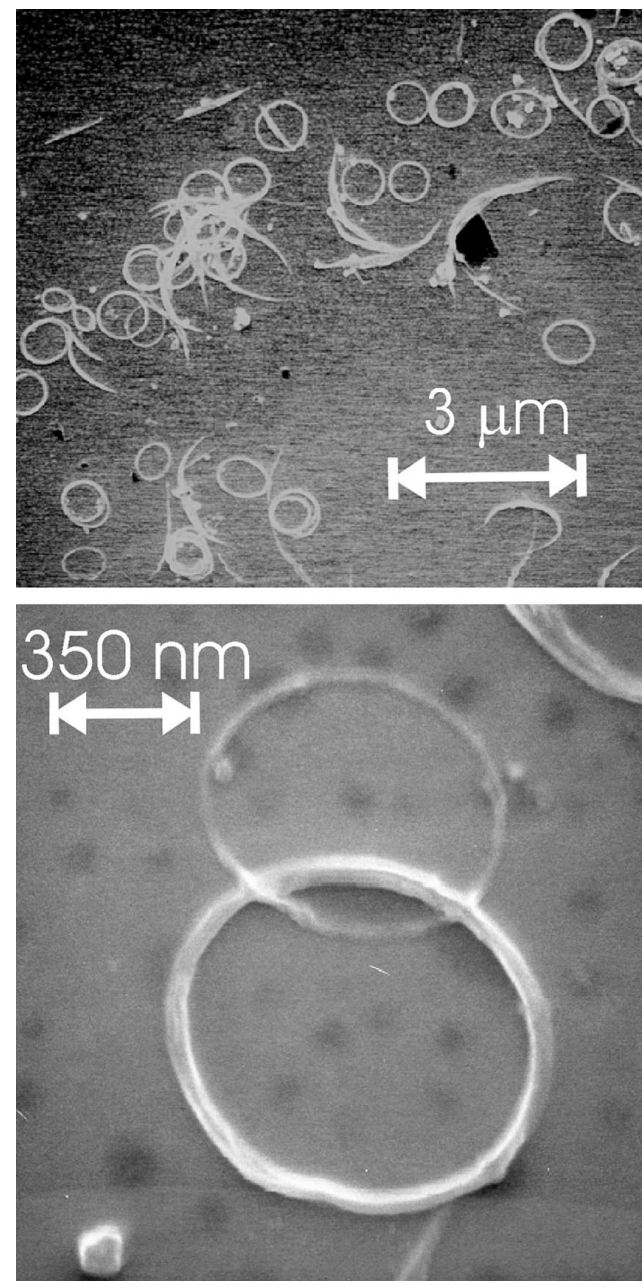

Fig. 5. Top: Scanning electron microscope images of nanotube rings produced from ropes of single-wall nanotubes. Bottom: Images of two inter-connected nanotube rings.

Some of the loops appear to be single circular tubes. However, by AFM manipulation they can be unwound, suggesting that there are no covalent bonds holding the ropes together. The loops are fairly large (about 400-800 $\mathrm{nm}$ radius). Therefore, the strain induced by the increased curvature can be compensated by the gain in adhesion between nanotubes. This coiling behavior is similar to that observed in proteins and other biomolecules, where hydrogen bonding is thought to provide the main force for coiling. In the case of carbon nanotubes, however, only van der Waals forces are present. 


\section{AFM manipulation of carbon nanotubes}

In order to use individual carbon nanotubes to build nanostructures and devices one must be able to manipulate them and place them at predefined positions at will. The interaction between the nanotubes and the substrate on which they are dispersed sug- gests that one can manipulate their position at room temperature by applying lateral forces of the appropriate magnitude with the tip of an AFM. We have found that the shear stress on surfaces such as H-passivated silicon is high, of the order of $10^{7} \mathrm{~N} / \mathrm{m}$, such that not only can the position of the nanotube be controlled but also their shape [8].
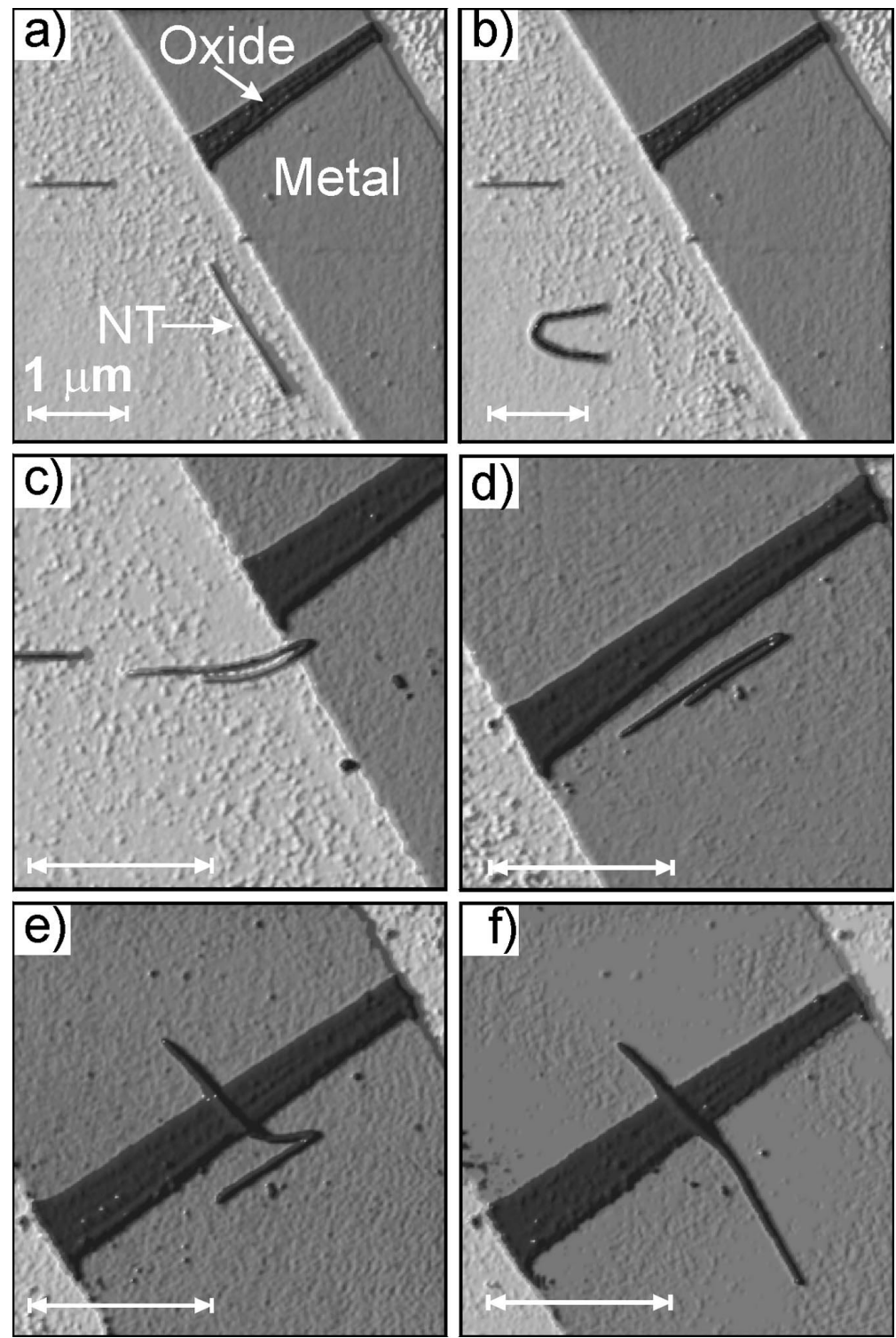

Fig. 6. AFM manipulation of a single multi-wall nanotube such that electrical transport through it can be studied. Initially, the nanotube (NT) is located on the insulating $\left(\mathrm{SiO}_{2}\right)$ part of the sample. In a stepwise fashion (not all steps are shown) it is dragged up the $80 \AA$ high metal thin film wire and finally is stretched across the oxide barrier. 
To manipulate the nanotubes, we have to change the mode of operation of the AFM. While for tube imaging we use the AFM in the non-contact mode with very low forces applied by the tip (in the range of $\mathrm{pN}$ ), the contact mode with normal forces of $10-50 \mathrm{nN}$ is employed for manipulation. In Fig. 6, we show a sequence of manipulation steps. A single nanotube originally on an insulating substrate $\left(\mathrm{SiO}_{2}\right)$ is manipulated in a number of steps (not all shown) onto a tungsten thin film wire ( $\sim 80 \AA$ high), and, finally, is stretched across an insulating $\mathrm{WO}_{x}$ barrier (itself made by AFM tip-induced oxidation [11] at an earlier stage). It is interesting to note that highly distorted tube configurations are formed during the manipulation process and stabilized by the interaction with the substrate. The ability to prepare locally highly strained configurations and the well known dependence of chemical reactivity on bond strain suggest that manipulation may be used to induce local chemistry.

\section{Electrical transport, single nanotube field-effect transistors, and Coulomb blockade}

Carbon nanotubes are thought to be examples of ideal one-dimensional quantized conductors [1]. Given the fact that electrons in $\pi$-states are involved in the conduction, the low bias resistance of the nanotube for ballistic transport should be $h / 4 e^{2}$, i.e., about $6 \mathrm{k} \Omega$ [12]. Electrical measurements on individual nanotubes became possible recently [1317], often by preparing electrical contacts, dispersing nanotubes on them and relying on chance that a nanotube will end up bridging the electrodes. As we saw in Section 3, AFM manipulation allows full control of this process. In Fig. 7, we show an AFM image of two gold electrodes and several multi-wall nanotubes dispersed on them. By AFM tip manipulation one of the nanotubes is placed such that it bridges the two electrodes and its $I-V$ characteristics can be evaluated. An ohmic behavior is seen in the
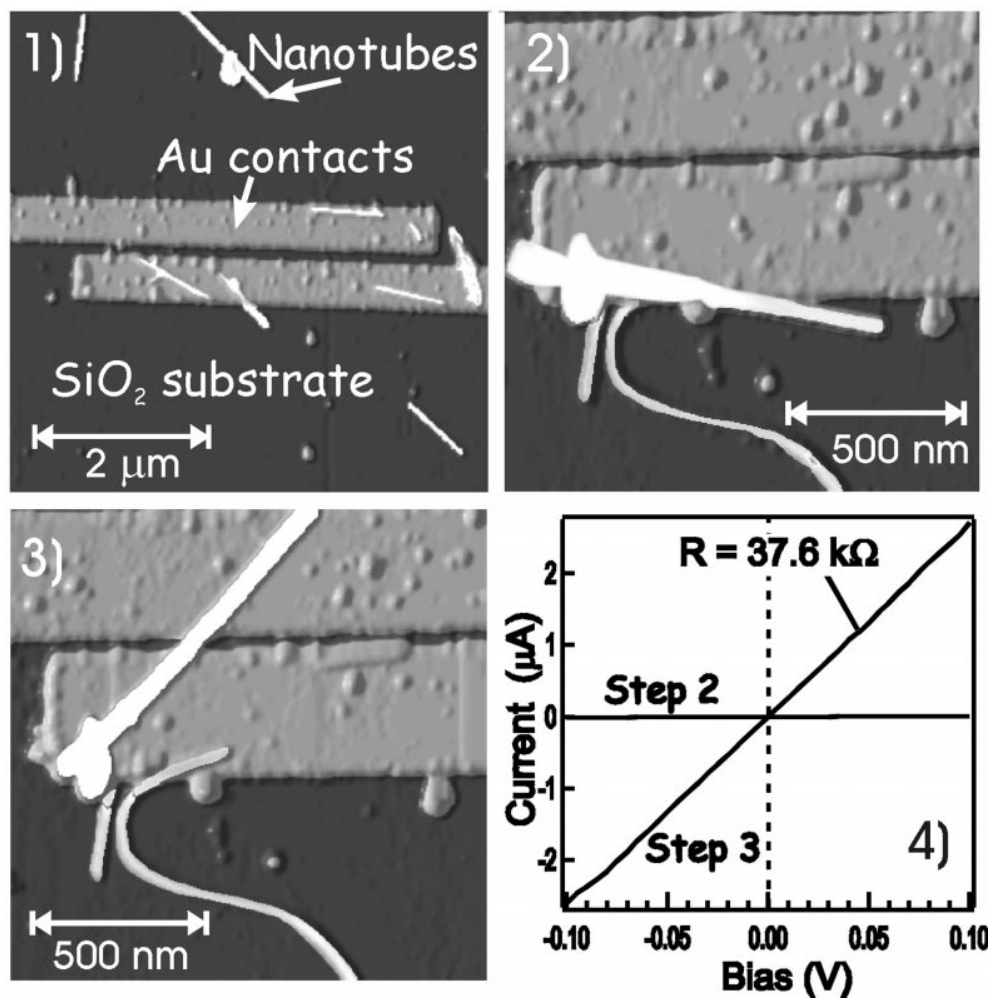

Fig. 7. (1) A pair of gold electrodes and a number of multi-wall nanotubes. (2) Close up of the electrodes and of a tube partially overlapping one of the electrodes. (3) The tube is manipulated so as to bridge the gap between the two electrodes. (4) The current flowing between the electrodes at step 2 and after step 3. 
bias range of $\pm 100 \mathrm{meV}$ from which a resistance of $38 \mathrm{k} \Omega$ is obtained. This resistance is higher than that expected for ballistic transport. However, experimentally even higher resistances of the order of 1 to 100 $\mathrm{M} \Omega$ are more commonly found. The $I-V \mathrm{~s}$ in the cases of high resistance are non-ohmic and suggest that the measured two probe resistance is dominated by tunneling through a barrier at the electrodenanotube contact. Our experiments showed that this contact resistance is very sensitive to contamination and roughness of the gold surface. It is particularly interesting that selective electron irradiation of the contact areas of such a device in the SEM is found to drastically improve the conductance. Fig. 8 illustrates this behavior. Initially, the low bias two-terminal resistance of this device was extremely high, $\sim 1$ $\mathrm{T} \Omega$, and the $I-V$ curve highly non-linear. After irradiation experiments with $25 \mathrm{keV}$ electrons delivering a total dose of $\sim 34 \mathrm{C} / \mathrm{cm}^{2}$, the resistance was reduced to $\sim 100 \mathrm{k} \Omega$ and the $I-V$ developed ohmic characteristics. It is important to note that the effect on the resistance is specific to the irradiation of the contact regions. Irradiation of the nanotube only does not change the resistance. Similar observations were made by Schönenberger et al. [18].
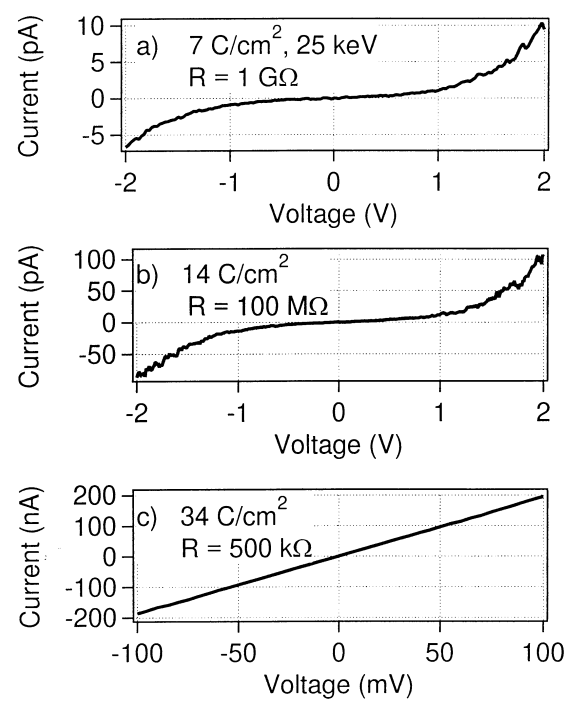

Fig. 8. The effects of irradiation by $25 \mathrm{keV}$ electrons on the current-voltage characteristics of the junction formed by a single nanotube bridging two gold electrodes. The initial resistance was $\sim 1 \mathrm{~T} \Omega$. The total dose after each irradiation is indicated on the graph.
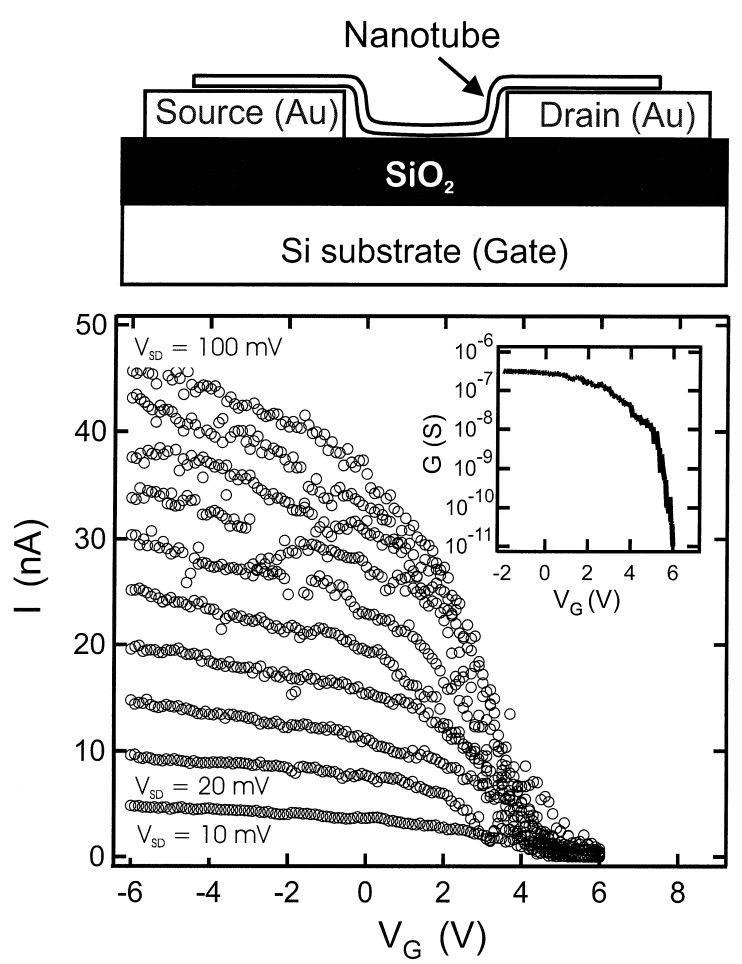

Fig. 9. A field-effect transistor based on a single nanotube. Top: A schematic of the device structure. Bottom: Source-drain current $I$ as a function of gate voltage $V_{\mathrm{G}}$ for several source-drain biases $V_{\mathrm{SD}}$. The inset shows the variation of the low-bias conductance $G=I / V_{\mathrm{SD}}$ of the nanotube as a function of the gate voltage.

For electrical applications, it is of great interest to know how much current can be passed safely through an individual nanotube. A coupling between the electrons and the vibrations of the nanotube would lead to energy dissipation, thus raising the temperature of the tube. For high enough currents the tube will be destroyed. Considering the multi-wall nanotube in Fig. 7, we found that it can carry a current of up to $60 \mu \mathrm{A}$ or a current density of the order of $10^{11}-10^{12} \mathrm{~A} / \mathrm{m}^{2}$ (depending on the actual area the current flows through). The tubes tend to break in the section which is suspended above the substrate between the electrodes, i.e., where heat dissipation is expected to be the lowest.

A very interesting development involves the fabrication of novel electronic devices that take advantage of the unique electrical properties of carbon nanotubes. Both room-temperature operation and a 
conventional switching mechanism such as in fieldeffect transistors (FETs) are highly desirable.

For this purpose we explored the possibility of using semiconducting tubes as channels in an FET configuration. As is shown schematically in Fig. 9 (top), a nanotube is positioned so as to bridge the gap between two gold electrodes defined lithographically on top of a $\sim 140 \mathrm{~nm}$ thick $\mathrm{SiO}_{2}$ film grown on a silicon wafer. The two gold electrodes can be viewed as the source and drain of the device, while the doped silicon wafer itself can be used as a back gate. Room-temperature results for a $\sim 16 \AA$ diameter single-wall nanotube are given in Fig. 9 (bottom) which shows the transfer characteristics of the device, i.e., the variation of the source-drain current $I$ as a function of the gate voltage $V_{\mathrm{G}}$ for several source-drain biases $V_{\mathrm{SD}}$. These results clearly demonstrate that the gate can strongly modify the current flow through the nanotube. The enhancement of the current at negative gate bias indicates that positive holes are the main carriers. Magnetoresistance measurements on nanotubes have reached the same conclusion [19]. The nanotube FET is a normally 'on' device which can be switched to the 'off' state by a positive gate bias. As the inset in Fig. 9 shows, the gate can modulate the low-bias conduc-

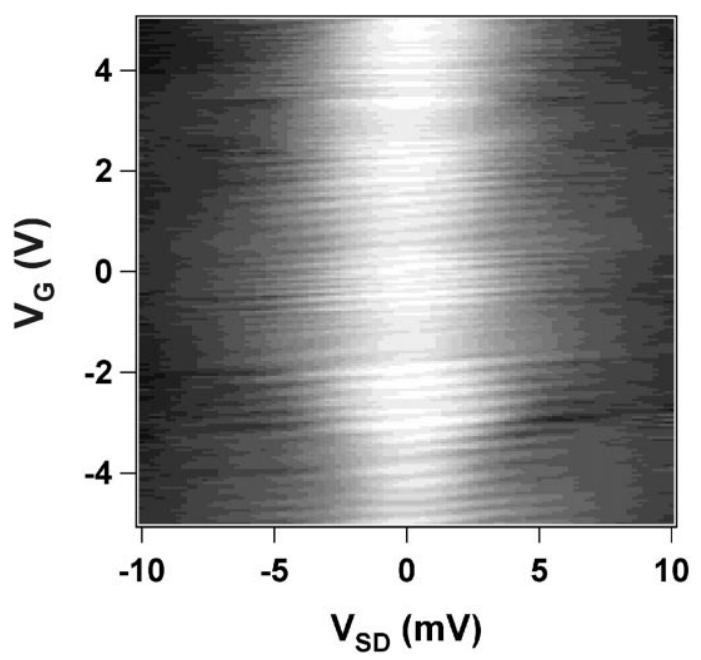

Fig. 10. Single-electron transistor operation using a bundle of single-wall nanotubes as Coulomb island. The differential conductance $\mathrm{d} I / \mathrm{d} V_{\mathrm{SD}}$ of the nanotube bundle is plotted as a function of source-drain voltage and gate voltage (white: low conductance; black: high conductance).

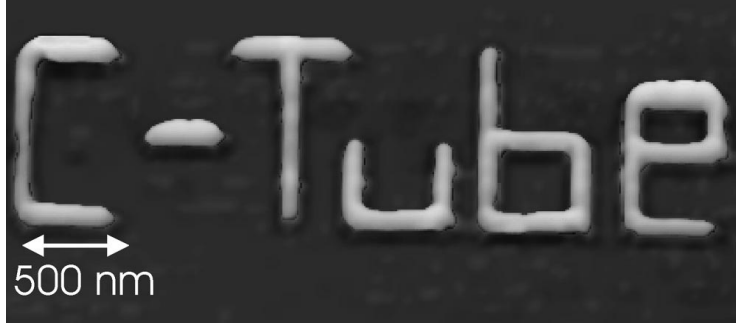

Fig. 11. Use of conducting carbon nanotube AFM tips as nanofabrication tools. In this case, a nanotube-bundle tip was used as the negative electrode to locally oxidize silicon and write the oxide pattern 'C-Tube'.

tance $G=I / V_{\mathrm{SD}}$ of the tube by 5 orders of magnitude. While this manuscript was in preparation, a paper by Tans et al. appeared which also reports the fabrication of an FET based on a single-wall nanotube [20]. The results of the two groups are in good agreement.

Large multi-wall nanotubes are, in principle, not expected to be useful for field-effect devices since the band gap of semiconducting tubes should decrease as $1 / d[21]$. Thus, at room temperature, such nanotubes should effectively behave as if they were metallic. However, as we discuss elsewhere [22], we have been able to observe an interesting gate effect in the case of a deformed multi-wall nanotube.

Using metallic nanotubes as Coulomb islands, single-electron transistor (SET) action has been demonstrated at low temperature [16,17]. Spreading bundles of single-wall nanotubes on electrode configurations such as the one sketched in Fig. 9 (top), we observed Coulomb blockade at liquid helium temperature. Fig. 10 shows a gray-scale plot of the differential conductance $\mathrm{d} I / \mathrm{d} V_{\mathrm{SD}}$ as a function of source-drain voltage and gate voltage. As a consequence of Coulomb blockade, rhombically shaped structures of vanishing current (white) occur periodically as the gate voltage is varied. The nanotube bundle is charged one by one with dozens of individual electrons as the device operates as a single-electron transistor. In the case of a single metallic island, the width of the Coulomb gap is independent of the gate voltage [23]. In our nanotube SET, the gap width oscillates as a function of the gate voltage, which is suggestive of single-electron transport through multiple Coulomb islands formed within the nanotube bundle. 


\section{Carbon nanotube tips as electrodes for local anodization of surfaces}

The unique structure, mechanical and electrical properties of carbon nanotubes make them promising materials for use as STM or AFM tips. Their shape allows them to probe crevices and image structures with large curvature gradients. Dai et al. [24] were the first to recognize this potential. We have found that the electrical conductivity of such tips makes them useful not only for STM imaging but also in device fabrication. In particular, they can be used as the negative electrode in nanoscale tip-induced anodization. In Fig. 11, we show an example where a bundle of multi-walled nanotubes is used as a tip to oxidize a H-passivated silicon surface and generate the oxide pattern ' $\mathrm{C}$-Tube'. For this purpose, the nanotube tip is biased at $-10 \mathrm{~V}$ while being scanned in contact with the surface under ambient conditions (relative humidity of $20 \%$ ). The oxidant is the atmospheric $\mathrm{H}_{2} \mathrm{O}$, which is condensed by capillary action near the apex of the tip. As discussed elsewhere in detail [25], $\mathrm{OH}^{-}$ions are driven by the strong field into the solid and induce the oxidation by reacting with Si holes in bulk Si. Although in this experiment the resolution obtained is comparable to that achieved with conventional tips, we believe that it can be enhanced significantly by using single-nanotube tips.

\section{Acknowledgements}

We thank A.G. Rinzler, R.E. Smalley and H. Dai for providing us with the single- and multi-wall nanotubes.

\section{References}

[1] M.S. Dresselhaus, G. Dresselhaus, P.C. Eklund, Science of Fullerenes and Carbon Nanotubes, Academic Press, San Diego, 1996.

[2] A. Thess et al., Science 273 (1996) 483

[3] R. Saito et al., Appl. Phys. Lett. 60 (1992) 2204.

[4] J.W.G. Wildöer et al., Nature 391 (1998) 59.

[5] T.W. Odom et al., Nature 391 (1998) 62.

[6] M.M.J. Treacy, T.W. Ebbesen, J.M. Gibson, Nature 381 (1996) 678.

[7] E.W. Wong, P.E. Sheehan, C.M. Lieber, Science 277 (1997) 1971.

[8] T. Hertel, R. Martel, Ph. Avouris, J. Phys. Chem. B 102 (1998) 910

[9] T. Hertel, R.E. Walkup, Ph. Avouris, Phys. Rev. B 58 (1998).

[10] R. Martel, H.R. Shea, Ph. Avouris, to be published.

[11] Ph. Avouris, T. Hertel, R. Martel, Appl. Phys. Lett. 71 (1997) 285

[12] L. Chico et al., Phys. Rev. B 54 (1996) 2600.

[13] L. Langer et al., Phys. Rev. Lett. 76 (1996) 479.

[14] H. Dai, E.W. Wong, C.M. Lieber, Science 272 (1996) 523.

[15] T.W. Ebbesen et al., Nature 382 (1996) 54.

[16] S.J. Tans et al., Nature 386 (1997) 474.

[17] M. Bockrath et al., Science 275 (1997) 1922.

[18] A. Bachtold et al., Appl. Phys. Lett. 73 (1998) 274.

[19] S.N. Song et al., Phys. Rev. Lett. 72 (1994) 697.

[20] S.J. Tans, A.R.M. Verschueren, C. Dekker, Nature 383 (1998) 49.

[21] V.H. Crepi, M.L. Cohen, A. Rubio, Phys. Rev. Lett. 79 (1997) 2093.

[22] R. Martel, T. Schmidt, H.R. Shea, T. Hertel, Ph. Avouris, Appl. Phys. Lett. 7 (1998) 2447.

[23] H. Grabert, M.H. Devoret (Eds.), Single Charge Tunneling: Coulomb Blockade Phenomena in Nanostructure, NATO ASI Series, Vol. 294, Plenum Press, New York, 1992.

[24] H. Dai et al., Nature 384 (1996) 147.

[25] Ph. Avouris et al., Appl. Phys. A 66 (1998) S667. 